\title{
Vigilance for pain-related faces in a primary task paradigm: an ERP study
}

\author{
This article was published in the following Dove Press journal: \\ Journal of Pain Research \\ 8 June 2013 \\ Number of times this article has been viewed
}

\author{
Stefan Lautenbacher' \\ Oliver Dittmar' \\ Corinna Baum ${ }^{1,2}$ \\ Raphaela Schneider ${ }^{\prime}$ \\ Edmund Keogh ${ }^{3}$ \\ Miriam Kunz' \\ 'Physiological Psychology, Otto- \\ Friedrich University Bamberg, \\ Bamberg, Germany; ${ }^{2}$ Institute of \\ Medical Psychology, Justus-Liebig \\ University Giessen, Giessen, Germany; \\ ${ }^{3}$ Centre for Pain Research and \\ Department of Psychology, University \\ of Bath, Bath, UK
}

Background: Pain-related stimuli are supposed to be automatically prioritized over other stimuli. This prioritization has often been tested using primary task paradigms in which pain information is irrelevant for completing the explicitly posed task. Task-irrelevant stimuli are only processed if they are very salient, and pain-related stimuli are assumed to be salient enough.

Objective: We wanted to further investigate this assumption by assessing event-related brain potentials (ERPs) - a very sensitive method for studying attention and reaction times in response to pictures of people in pain and other emotional faces - using a primary task paradigm. In addition, we assumed that individuals describing themselves as vigilant to pain are especially responsive to pain cues.

Methods: One hundred pain-free subjects were tested in a primary task paradigm using pictures of facial expressions of pain, anger, happiness, and neutral mood. ERPs were assessed at midline electrodes. Vigilance to pain was assessed by the pain vigilance and awareness questionnaire.

Results: In contrast to previous studies (which have used pain words), effects of facial expressions of pain and other emotions on the ERPs and reaction times were surprisingly weak throughout and did not give evidence for a distinct processing of pain-related stimuli. However, hypervigilant subjects appeared to be strongly and cognitively absorbed by all emotional stimuli.

Conclusion: Accordingly, it appears that pain-related stimuli are not always of outstanding salience, but that certain characteristics (eg, type of material, emotional richness) have to be present, for pain-related stimuli to be prioritized over stimuli of other emotional content. Hypervigilance to pain may generally predispose individuals to process emotional stimuli in greater depth.

Keywords: hypervigilance for pain, ERP, LPC, pain face, primary task

\section{Introduction}

Vigilance to pain is defined as automatic and unintentional processing of pain-related stimuli at the cost of competing stimuli and is assumed to occur because of the outstanding salience of pain-related stimuli. ${ }^{1}$ Such vigilance guarantees that, even under disadvantageous conditions, pain as a biologically relevant stimulus is rarely missed.

The question arises of whether only pain itself or already pain-related cues meet these assumptions. A strong similarity between "true pain" and pain-related cues has been suggested by imaging data, which showed that pain symbols - like words produce similar bold cerebral reactions as do noxious stimuli. ${ }^{2}$ It might be argued that the study of pain-related cues or symbols, in the absence of "true" physical pain, is even more informative for certain diagnostic purposes. If cues associated with pain
Correspondence: Stefan Lautenbacher
Physiological Psychology,
Otto-Friedrich University
Bamberg, Markusplatz 3,
96045 Bamberg, Germany
Tel +49 95I 863 I85 I
Fax+49 95I 863 I976
Email stefan.lautenbacher@uni-bamberg.de 
are able to preferentially capture attention because of their salience, such cues may be especially sensitive for singling out pain-hypervigilant individuals, since actual pain can be expected to capture and preferentially guide attention in all and not only hypervigilant individuals. ${ }^{3}$

The dot-probe task is one of the best-known examples of attention tests for pain-related cues, originally developed for use in anxiety research. ${ }^{4}$ Keogh et al, ${ }^{5}$ applying pain-related, emotional, and neutral words as stimuli in a dot-probe task, found more attentional engagement with pain words in individuals with high fear of pain. Similarly, strong attentional focus on physical threat was found in individuals with high levels of physical anxiety sensitivity. ${ }^{6}$ However, in chronic pain patients, attentional bias towards pain has not consistently been found. ${ }^{7-9}$ Other studies could also not replicate attentional absorption by pain-related words in healthy subjects with high fear of pain. ${ }^{10,11}$ Accordingly, the use of words in the dot-probe task has not yet produced unequivocal findings.

More recent studies on chronic pain patients found attentional biases towards facial expressions of pain ${ }^{12}$ and towards headache-related pictures. ${ }^{13}$ Potentially, pictorial pain descriptors are more ecologically valid stimuli than verbal ones and might therefore be more suitable for detecting hypervigilance. In a previous study carried out by our group, Baum et $\mathrm{al}^{14}$ demonstrated, in a modified dot-probe task, higher early attentional engagement with pain faces and stronger later attentional avoidance of the same faces in individuals with high fear of pain (vigilance-avoidance pattern of attention).

The dot-probe task is a primary task paradigm, in which the pain-related stimuli are task-irrelevant (given that the subject is asked to respond to the position of a dot and not to the pain-related stimuli). This approach is sensitive to the attentional capture of very salient stimuli because only such stimuli are still traceably processed if completely taskirrelevant. The dot-probe task is based on reaction time measurement, which provides summary information about stimulus processing. However, reaction time measurement does not allow for an easy differentiation between the various stages of information processing. Disentangling stages of processing becomes possible by studying temporally separate components of event-related brain potentials (ERPs). Therefore, it might be favorable to adjust primary task paradigms to the requirements of ERP assessment.

There are already examples of this; utilizing verbal material, Nikendei et $\mathrm{al}^{15}$ found enhanced positive deflections between 350-600 milliseconds (P300) and higher late positive slow wave amplitudes between 500-800 milliseconds (late positive complex [LPC]) for pain-related compared to neutral words in a lexical decision task, a finding which was replicated by our research group. ${ }^{16}$ It has not yet been examined whether these effects are specific to word material or can also be shown for pictorial pain cues.

In the present study we attempted to assess ERPs for pain-related cues utilizing facial expressions of pain and other emotions (angry, happy, and neutral) in a visual primary task paradigm. Anger and joy were added to present very similar and very dissimilar displays of emotions, respectively, as a test of the specificity of the pain findings. We hypothesized effects for P300 and LPC under the assumption that the earlier findings obtained with word material can be generalized to pictorial stimuli. Furthermore, we expected these effects to be most prominent in individuals who are supposed to have habitually strong attentional biases regarding pain-related stimuli, namely those with high levels of pain vigilance. Our decision to study the effects of pain vigilance ahead of other similar concepts such as pain catastrophizing and pain anxiety was due to the model proposed by Goubert et $\mathrm{al}^{17}$ in which vigilance was claimed to be more directly related to pain experience than the other concepts, and to our previous findings of pain vigilant subjects being particular experts in pain face recognition. ${ }^{18}$

\section{Materials and methods Subjects}

A total of 100 healthy subjects between the ages of $18-65$ years ( mean $=39.7$ years, standard deviation $[\mathrm{SD}]=13.4$ years; 50 females, 50 males, no age differences between sexes) and equally distributed in four age categories (18-29, 30-39, 40-49, 50-65 years) were recruited via announcement in newspapers, public buildings in Bamberg, and among students of the Otto-Friedrich University of Bamberg. Subjects had normal or corrected-to-normal eyesight. Preliminary to testing, all subjects were screened by trained psychologists using stringent and standardized exclusion criteria: history of any psychiatric (assessed with SCID-I, a structured clinical interview according to Diagnostic and Statistical Manual of Mental Disorders-IV) ${ }^{19}$ or neurological disorders, substance abuse, psychopharmacological treatment, use of analgesics, acute or chronic pain, previous major surgical intervention, or prosopagnosia. The pain history was a special focus of the interview and was addressed by asking the participants separately for acute and chronic pain experience (current and past pain) and checking for potentially painful physical conditions (migraine, back pain, rheumatoid arthritis, etc). 
Based on the SCID, one participant was excluded from the study (schizophrenic disorder: F20.3). Subjects were asked to refrain from smoking and ingesting caffeine for 1 hour prior to testing because nicotine and caffeine could have an effect on attentional performance. Twenty-eight of the participants had a university degree, and 64 participants had a high school degree (29 subjects out of the 64 were students). Sixty-five of the participants lived with a partner. Except for students who participated to fulfill course requirements, all subjects were paid $40 €$ for attendance. All subjects gave informed consent and were free to terminate the experiment at any time. The study was approved by the Ethical Committee of the University of Bamberg.

\section{Procedure}

The sessions took place between 9 am and $6 \mathrm{pm}$ and lasted for approximately 1 hour. On arrival at the laboratory, subjects were informed about the course and duration of the experiment, asked to sign the informed consent form, and to fill out a questionnaire regarding their ability to recognize and imagine faces. ${ }^{20} \mathrm{We}$ included this measure to allow for the exclusion of clinical states and to control for possible subclinical states of prosopagnosia.

For recording the electroencephalogram (EEG) electrodes were attached. Subjects were seated in front of a 20-inch computer screen (1 meter viewing distance) which was used to present the experimental task. The experimenter gave instructions verbally and left the room before the start of testing. Subjects were monitored through a video camera. If further interaction between experimenter and subject was needed during the test, it was done through a two-way communication system. At the end of the session, the subjects filled out the questionnaire, which was designed to target pain vigilance.

\section{Experimental paradigm: visual discrimination}

Ninety-six black and white pictures of faces with four emotional categories (anger, happy, neutral, and pain; 24 pictures for each category), of which $50 \%$ were overlaid with a small grid (12 with and 12 without grid in each emotional category), were presented in randomized order. Characteristics and selection of the pictures will be described in detail below.

As we intended to examine unintentional (ie, task-irrelevant) processing of pain-cues in this study, we presented the pictures in a primary task (ie, a visual discrimination of presence or absence of the grid). As no particular instructions were given regarding the pictures' contents, processing of the content - pain-related and other emotional faces - was irrelevant for successfully completing the primary task. Subjects were instructed to indicate in each trial whether a picture with or without a grid was presented by pressing one of two response buttons as fast as possible. Reaction times and accuracy were recorded.

Each single trial started with the question "Gitter?" (English: grid) presented for 700 milliseconds, reminding the subject of the upcoming task (ie, to decide whether the presented picture was overlaid with a grid) (see Figure 1). Then the picture (with or without a grid) was presented for 300 milliseconds and subjects had to press one of two response buttons, either the "grid" or the "non-grid" button. Immediately following the response a white bar was presented on the screen, paralleled by a continuous tone of 1000 hertz $(\mathrm{Hz})$ for 1500 milliseconds. Subjects had been instructed to use this interval for eye-blinks and to abstain from blinking for the rest of the trial; this was intended to minimize eyeblinks during relevant periods of data acquisition. The trial sequence ended with a black screen for 1000 milliseconds serving as inter-trial interval (see Figure 1). Subjects were familiarized with the procedure by running through ten training trials.

The primary task was designed to be of medium difficulty and was tested first in a pilot study. Concurrently presented stimuli relating to the secondary task should be able to capture attention if they are perceived as being strongly salient to the individual. Too difficult a primary task would prevent shifting to the secondary task completely; whereas too easy a primary task would be automatically processed after a

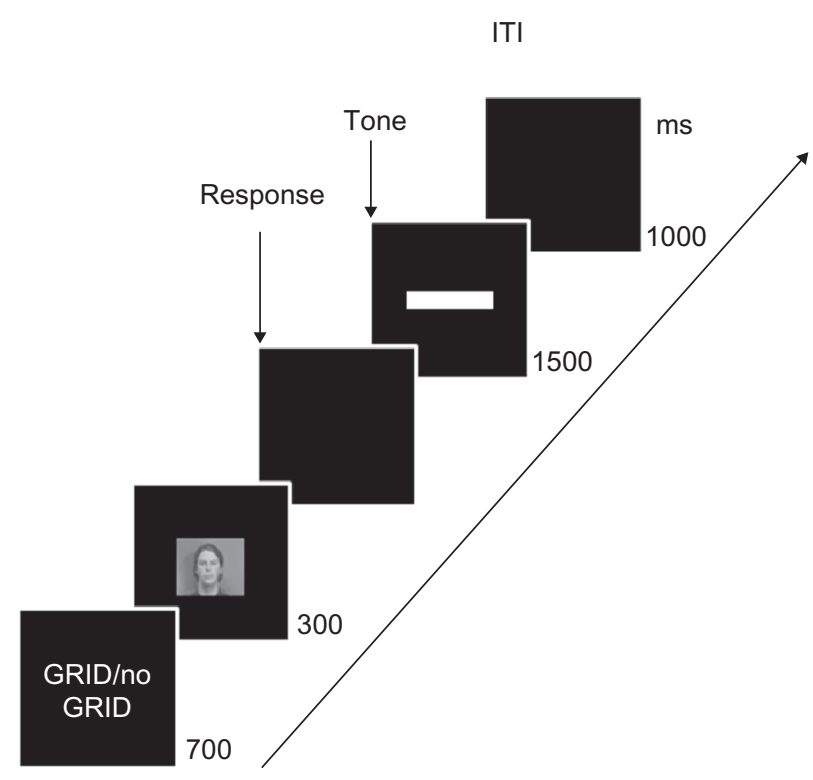

Figure I Sequence of experimental stimuli (trial format). Abbreviations: ITI, Interstimulus interval; ms, milliseconds. 
short while allowing constant shifts to the secondary task. The error distribution proved that the primary task was of medium difficulty; only a few subjects $(15.2 \%)$ were without any error, the majority of subjects $(75.0 \%)$ produced one to seven errors in 96 trials and again only a few subjects $(9.8 \%)$ produced a larger amount of errors (eight or more) (these error data are from 92 subjects, who remained for analysis after EEG artifact rejection; see description below).

\section{Stimulus material}

We generated 96 black and white pictures with 24 painful, 24 happy, 24 angry and 24 neutral facial expressions from the Montréal pain and affective face clips,${ }^{18,21}$ which is a collection of one-second-videos of eight professional actors (four females) mimicking affective and painful facial expressions. The actors were all Caucasians, as were our subjects. For each expression type, five different snapshots were extracted, resulting in a set of 160 pictures (four types of expressions $\times$ five snapshots $\times$ eight actors). Before applying these stimuli in the present study on attentional processing, we validated this material. For that purpose we had 40 subjects categorize these pictures as belonging to one of the four different facial expressions. A ranking of the pictures was made according to hit-rate (eg, a pain-face is correctly categorized as pain), rejection rate (eg, a pain-face is not categorized as any of the other emotional expressions), and reliability. ${ }^{18}$ The 96 topranking pictures were chosen for the present study.

\section{Reaction time}

As stated above, subjects were instructed to indicate in each trial whether a picture with or without a grid was presented by using a response box (for a more detailed description of the reaction time measurement see Baum et al). ${ }^{14}$ In the present study, we used a three-button response panel. A centrally positioned button served as a holding button and the two other buttons (slightly above and either to the right or to the left of the holding button) were used to indicate whether a grid (right button) or no grid (left button) were presented. Subjects practiced in a few training trials until they were able to selectively press the response buttons for grid and no-grid trials. In each trial, the starting position of the index finger was on the central holding button to reduce variance in movement distances to the response buttons. Participants were instructed to release the holding button only after appearance of the picture to indicate whether it was overlaid with a grid or not. Reaction time was taken from the time interval between appearance of a picture and the release of the holding button (once a decision had been made).

\section{Physiological recording}

An EEG was recorded from three midline sites (frontal at Fz; central at $\mathrm{Cz}$; and parietal at $\mathrm{Pz}$ ) and $\mathrm{A} 1 / \mathrm{A} 2$ (mastoid) with a commercially available electrode cap (Electro-Cap International, Inc, Eaton, OH, USA) with tin electrodes placed according to the international $10-20$ system. ${ }^{22}$ For measuring the vertical electrooculogram (EOG), tin electrodes were placed above and below the right eye; for measuring horizontal electrooculogram, tin electrodes were placed at the outer canthi. The EEG, including vertical and horizontal EOG, was continuously recorded with a DC Brain Amp amplifier (Brain Products GmbH, Gilging, Germany) with a sampling rate of $1000 \mathrm{~Hz}$, a notch-filter at $50 \mathrm{~Hz}$, and a low pass filter at $70 \mathrm{~Hz}$. Off-line, data was filtered with a $30 \mathrm{~Hz}$ low pass filter, and a $0.1 \mathrm{~Hz}$ high pass filter. All channels were primarily recorded with a $\mathrm{Cz}$ reference. Off-line, data was re-referenced to linked A1/A2, whereby Cz was regained for further analysis. Sweeps of 1000 millisecond duration, starting at 200 milliseconds before stimulus onset, were extracted and adjusted to the 50 millisecond baseline-period just before stimulus onset. Further, data was corrected for EOG artifacts using a regression method. ${ }^{23}$ Elimination of sweeps with artifacts was done by automatically detecting sweeps with amplitudes outside a range of \pm 80 microvolts and with gradients over 100 microvolt change per sampling point. ${ }^{24-27}$ Further, manual single-trial visual inspection was done excluding sweeps with uncorrected eye-blinks, dominant alpha waves, and generally distorted sweeps due to high-frequency noise. Averaged event-related potentials (ERPs) for the four emotional categories in both the grid or no-grid conditions were calculated. Averages were only calculated if at least 8 out of 12 sweeps per condition were accepted in the artifact-rejection procedure. If in one or more experimental conditions no averages could be calculated, the corresponding subject was excluded. The described rigorous procedure of artifact rejection forced us to exclude eight subjects, so that a sample of 92 subjects remained.

For the LPC, which has previously been examined in primary task paradigms using verbal stimuli, ${ }^{15,16,27}$ we distinguished - in accordance with the literature ${ }^{28-32}$ and with our visual inspection of the graphs with grand averages between an early activation at 260-460 milliseconds (late positive complex 1 [LPC1]) and a late activation at 460-800 milliseconds (late positive complex 2 [LPC2]). These potentials were measured as mean amplitudes because no clear peak could be discerned in this time range.

In addition, we extracted positive peaks in a time range from 260-400 milliseconds after stimulus-onset (P300), 
which has also been previously reported from primary task paradigms including pain-related words as a critical component. ${ }^{15,16}$ Further, we extracted averaged peak amplitudes of an earlier positive deflection in the time range of 170-230 milliseconds after stimulus-onset (P200), which has been referred to as being modulated by emotional/pain-related content in passive viewing paradigms without task requirements. ${ }^{33,34}$

\section{Pain hypervigilance}

Since we were interested in whether the responses to the emotional face expressions might be influenced by the subjects' tendency to be vigilant to pain, subjects were asked to complete a validated German version of the pain vigilance and awareness questionnaire (PVAQ). ${ }^{35}$ The PVAQ was developed as a comprehensive measure of attention to pain and has been validated for the use in chronic pain and non-clinical samples. It consists of 16 items that are rated on a six-point scale assessing awareness, vigilance, preoccupation, and observation of pain. For further analyses, we used the median score, dividing subjects into a group of "low" and "high" vigilant individuals (the median score in the present sample was 36).

\section{Data reduction and statistics}

We expected medium effect sizes (Cohen's $d=0.50$ ) for power analysis (Sample Power; SPSS, IBM Corporation, Armonk, NY, USA). The result showed that a sample size of 50 or more provides strong power at the significance level at 0.05 for within-subjects analyses. We, however, included more subjects to be able to also calculate between-subjects analyses (high and low pain vigilant individuals).

For evaluation of the reaction times, we calculated a repeated-measures ANOVA with the within-subject factors, emotion (anger, happy, neutral, and pain) and grid (with or without grid), and the between-subject factor, vigilance (PVAQ; "low" and "high" vigilance to pain).

For the averaged ERPs of the components P200, P300, LPC1, and LPC2, we calculated a repeated-measure ANOVA with the within-subject factors, emotion (anger, happy, neutral, and pain), grid (with or without grid), and topography (frontal, central, and parietal), and the between-subject factor, vigilance ("low" and "high" vigilance to pain). The main focus of our analyses was on the effects of the different emotions on the ERPs. Therefore, not all main and interactions effects were subjected to fine-grain post hoc analyses. However, if in any of the ANOVAs a main-effect or an interaction, including the factor emotion, were found to be significant, we performed post hoc tests and used analyses of variance as well as $t$-tests for dependent samples to compare each emotion category with each other (anger versus happy, anger versus neutral, anger versus pain, happy versus neutral, happy versus pain, neutral versus pain). When violation of sphericity occurred in the ANOVAs, we used the Greenhouse-Geisser correction. We set the significance value as $P \leq 0.05$.

\section{Results \\ Sample characteristics}

The described procedure of artifact rejection reduced the sample size from $\mathrm{n}=100$ to $\mathrm{n}=92$ (age: mean $=39.7$ years; $\mathrm{SD}=13.5$ years; range $=19-65$ years; sex: 47 female, 45 male). Of the female subjects, 39\% used contraceptives, $17 \%$ were in the first third of their menstrual cycle (day 1 to day 8 after cycle-onset according to self-report), $6 \%$ were in the second third (day 9 to day 17 ), $17 \%$ were in the third third (after day 17 ), and $21 \%$ were post-menopausal.

\section{Reaction time}

Analysis of variance revealed a main effect for the factor, grid $(\mathrm{F}[1,90]=74.230 ; P<0.001)$, with reaction times being significantly prolonged in the no-grid trials (see Figure 2). However, the factor, emotion, yielded no significant main effect on reaction times $(\mathrm{F}[3,270]=1.904 ; P=0.129)$, but emotion did interact significantly with the factor, grid $(\mathrm{F}[3,270]=2.871 ; P=0.039)$. As post hoc tests revealed, this interaction was due to the fact that emotion elicited a significant change of reaction times only in the grid trials $(\mathrm{F}[3,273]=4.614 ; P=0.005)$ but not in the non-grid trials $(\mathrm{F}[3,273]=1.490 ; P=0.363)$. More precisely, in the grid trials, subjects showed longer reaction times if a neutral or an anger face was presented compared to happy and pain faces (all $P$-values of $t$-tests for dependent samples comparing neutral/anger versus happy/pain were $<0.05$ ).

Interestingly, we also found a significant between-subject effect for vigilance $(\mathrm{F}[1,90]=5.593 ; P=0.020)$, with individuals scoring high on the PVAQ having prolonged reaction times (Figure 2). However, none of the interactions, including vigilance, yielded any significant effect.

\section{Analyses of variance of ERP data}

All within-subject main effects and interactions of the analyses of variance for the different ERP components are shown in Table 1. The impact of the between-subject factor, vigilance, (PVAQ score) is not shown in Table 1 for the sake of clear arrangement, but will be reported in the text. 


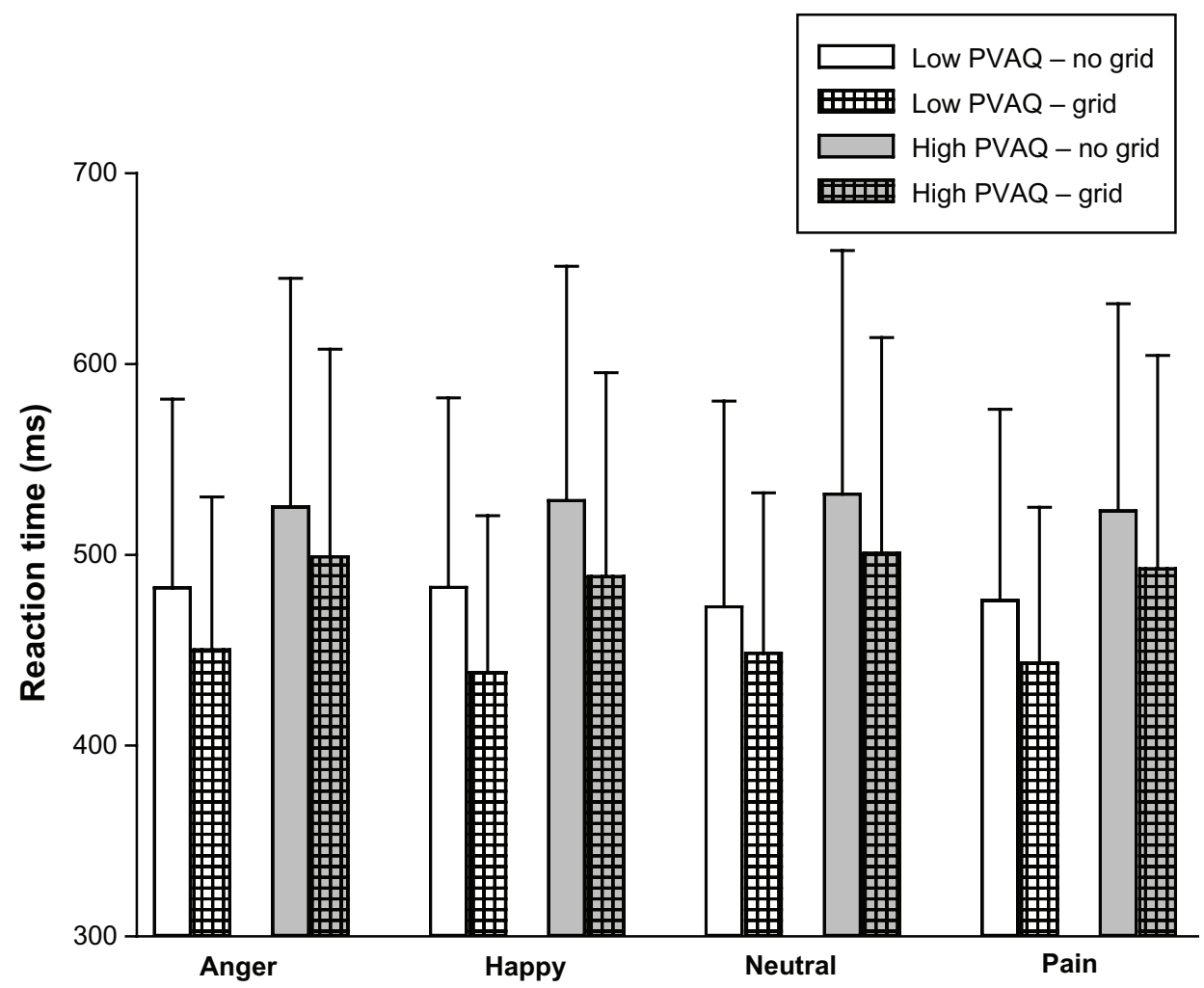

Figure 2 Mean and SD of reaction times for the four different types of emotional face expressions in trials with and without overlaid grid, for low and high pain vigilant subjects.

Abbreviations: ms, milliseconds; PVAQ, pain vigilance and awareness questionnaire; SD, standard deviation.

Grand averages are presented for the four categories of emotion in Figure 3A (Fz), 3B (Cz) and 3C (Pz).

\section{P200}

There were significant main effects for the factors, grid and topography, and for their interaction (Table 1). However, the most relevant main effect of the factor, emotion, remained insignificant as well as the interactions including this factor (Table 1). Furthermore, the between-subject factor, vigilance, also had no significant main effect $(\mathrm{F}[1,90]=0.002 ; P=0.967)$ nor interactive effects on P200 (all $P$-values $>0.05$ ). Therefore, no further post hoc analyses were conducted.

\section{P300}

Besides significant main effects for the factors, topography and grid, as well as a significant interaction of these two factors, the factor, emotion, produced a significant main

Table I All main within-subject effects and interactions of the analysis of variance with the factors, topography, grid, and emotion (F-value, $P$-value)

\begin{tabular}{|c|c|c|c|c|c|c|c|}
\hline $\begin{array}{l}\text { ERP } \\
\text { component }\end{array}$ & Topography & Grid & Emotion & $\begin{array}{l}\text { Topography } \times \\
\text { Emotion }\end{array}$ & $\begin{array}{l}\text { Topography } \times \\
\text { Grid }\end{array}$ & Grid $\times$ Emotion & $\begin{array}{l}\text { Topography } \times \\
\text { Grid } \times \text { Emotion }\end{array}$ \\
\hline \multirow[t]{2}{*}{ P200 } & $F=12.758$ & $F=9.297$ & $F=0.868$ & $F=1.194$ & $F=17.350$ & $F=0.487$ & $F=0.358$ \\
\hline & $P<0.001$ & $P=0.003$ & $P=0.458$ & $P=0.308$ & $P<0.001$ & $P=0.692$ & $P=0.905$ \\
\hline \multirow[t]{2}{*}{ P300 } & $F=4 I .265$ & $F=65.356$ & $F=3.631$ & $F=1.48 I$ & $F=6.149$ & $F=2.405$ & $F=0.258$ \\
\hline & $P<0.001$ & $P<0.001$ & $P=0.014$ & $P=0.207$ & $P=0.003$ & $P=0.068$ & $P=0.956$ \\
\hline \multirow[t]{2}{*}{ LPCI (260-460) } & $F=52.109$ & $F=49.735$ & $F=1.123$ & $F=1.244$ & $\mathrm{~F}=3.30 \mathrm{I}$ & $F=2.285$ & $F=0.662$ \\
\hline & $P<0.001$ & $P<0.001$ & $P=0.340$ & $P=0.282$ & $P=0.059$ & $P=0.079$ & $P=0.612$ \\
\hline \multirow[t]{2}{*}{ LPC2 (460-800) } & $F=44.159$ & $\mathrm{~F}=1.154$ & $F=1.635$ & $F=1.197$ & $F=0.105$ & $F=1.125$ & $F=1.724$ \\
\hline & $P<0.001$ & $P=0.286$ & $P=0.182$ & $P=0.306$ & $P=0.900$ & $P=0.339$ & $P=0.113$ \\
\hline
\end{tabular}

Notes: Significant findings are marked in bold; the tests with critical relevance for the hypotheses are shaded grey. In case of violation of the assumption of sphericity, the $P$-values are presented according to the Greenhouse-Geisser correction.

Abbreviation: ERP, event-related brain potentials; LPC, late positive complex. 


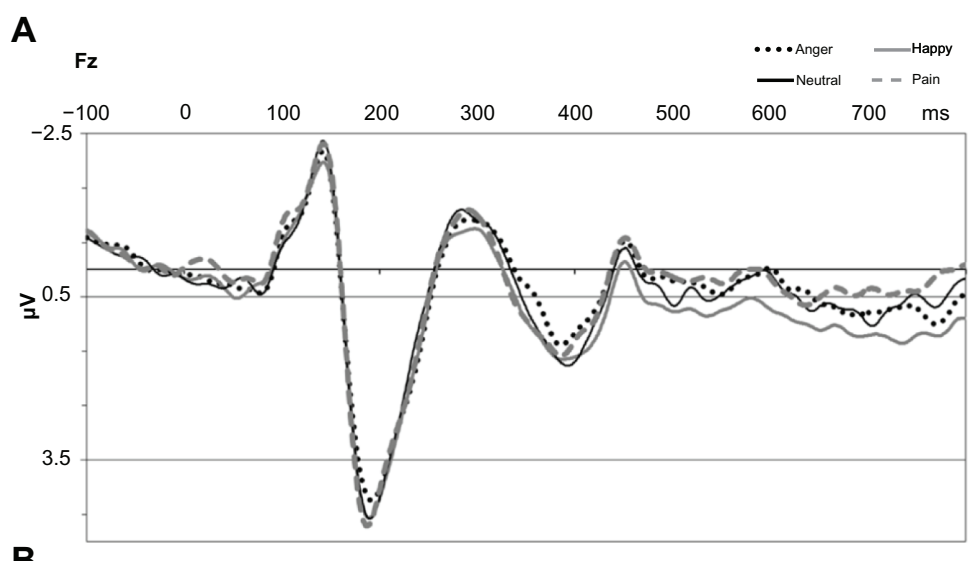

B
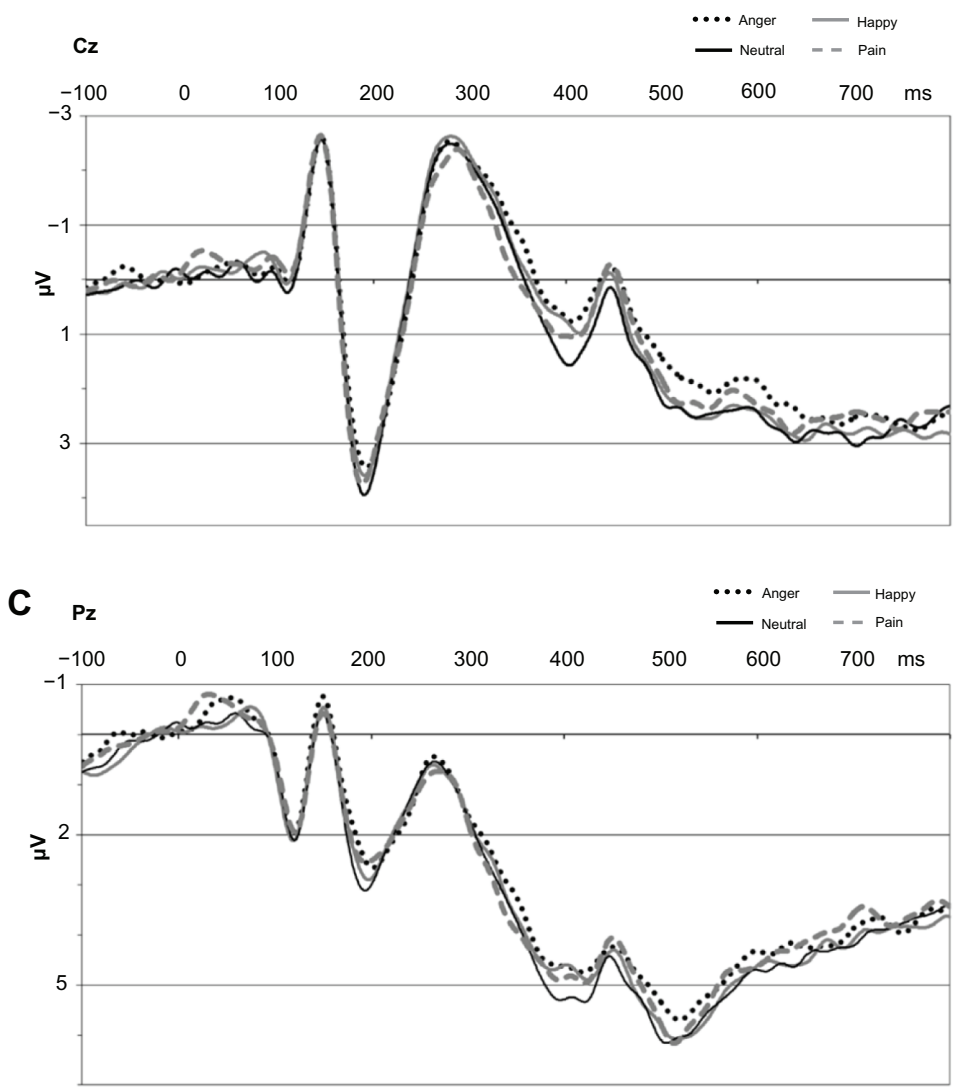

Figure 3 Grand averages for the four different categories of emotion at $\mathrm{Fz}, \mathrm{Cz}$, and $\mathrm{Pz}$.

Abbreviations: $\mathrm{Cz}$, central site; $\mathrm{Fz}$, frontal site; $\mathrm{Pz}$, parietal site; ms, milliseconds.

effect (Table 1). For the different categories of emotion, neutral faces were associated with the largest amplitudes, followed by pain, happy and anger faces; the latter producing the lowest amplitudes (Table 2). This impression was supported by post hoc $t$-tests for dependent samples (comparing P300 amplitudes averaged across the different topographies and across grid and no-grid trials between the different emotion categories), which revealed that anger faces elicited significantly lower amplitudes compared to neutral $(t[91]=-3.592 ; P=0.001)$ and compared to pain faces $(t[91]=-2.015 ; P=0.047)$. All other $t$-test comparisons revealed insignificant differences $(P$-values $>0.05)$. There was also a trend toward a significant interaction between emotion $\times$ grid (Table 1 ); post hoc analyses suggested that the different emotional face expressions only led to different $\mathrm{P} 300$ responses in the condition with overlaid grid $(\mathrm{F}[3,273]=4.367 ; P=0.0047)$, with anger faces again producing the lowest amplitude; whereas P300 responses did not differ between emotions in the no-grid trials $(\mathrm{F}[3,237]=1.706 ; P=0.167)$. 
Table 2 Mean and SD of the different ERP components (averaged across frontal, central, and parietal midline sites) for the four categories of emotion and for the presentations with and without grid

\begin{tabular}{lllll}
\hline & $\begin{array}{l}\text { Anger } \\
\text { Mean (SD) }\end{array}$ & $\begin{array}{l}\text { Happy } \\
\text { Mean (SD) }\end{array}$ & $\begin{array}{l}\text { Neutral } \\
\text { Mean (SD) }\end{array}$ & $\begin{array}{l}\text { Pain } \\
\text { Mean (SD) }\end{array}$ \\
\hline P200 & & & & \\
No grid & $4.98(3.0)$ & $5.11(3.4)$ & $5.17(3.1)$ & $5.01(3.4)$ \\
Grid & $5.19(3.2)$ & $5.63(3.1)$ & $5.56(3.2)$ & $5.66(3.6)$ \\
P300 & & & & \\
No grid & $3.42(3.5)$ & $3.36(3.3)$ & $3.85(3.5)$ & $3.36(3.4)$ \\
Grid & $4.41(3.6)$ & $4.93(3.5)$ & $5.20(3.5)$ & $5.28(4.2)$ \\
LPCI & & & & \\
No grid & $0.48(3.1)$ & $0.43(3.2)$ & $0.64(3.1)$ & $0.35(3.1)$ \\
Grid & $1.34(3.3)$ & $1.67(3.3)$ & $1.64(3.2)$ & $1.84(3.8)$ \\
LPC2 & & & & \\
No grid & $2.55(3.2)$ & $2.75(3.3)$ & $2.71(3.3)$ & $2.23(3.3)$ \\
Grid & $2.1 I(3.2)$ & $2.64(3.4)$ & $2.40(3.4)$ & $2.40(3.7)$ \\
\hline Abbrevat)
\end{tabular}

Abbreviations: SD, standard deviation; LPC, late positive complex.

Furthermore, the between-subject factor, vigilance, had a significant main effect on the $\mathrm{P} 300(\mathrm{~F}[1,90]=6.217 ; P=0.014)$, with pain vigilant subjects displaying overall increased $\mathrm{P} 300$ responses ("high" PVAQ: mean $=5.0$ microvolt $[\mathrm{SD}=3.2]$ versus "low" PVAQ: mean $=3.4$ microvolt $[\mathrm{SD}=2.8]$ ). However, these increased P300 amplitudes were not specific for any type of facial expression, given that vigilance did not interact with emotion or any of the other within-subject factors (all $P$-values $>0.05$ ).

\section{LPCI}

There was only significance for the main effects of the factors, topography and grid (Table 1). In contrast, the most relevant main effect of the factor, emotion, remained insignificant. However, as with the P300, there was a trend towards a significant interaction, emotion $\times$ grid. Post hoc analyses suggested again that different emotional face expressions only led to different LPC1 responses in the condition with overlaid grid $(\mathrm{F}[3,273]=2.980 ; P=0.049)$, again with anger faces producing the lowest amplitude; whereas responses did not differ between emotions in the no-grid trials $(\mathrm{F}[3,273]=0.536 ; P=0.658)$.

The impact of the between-subject factor, vigilance, on the LPC1 component just missed the level of significance $(\mathrm{F}[1,90]=3.726 ; P=0.057)$ and did not interact with any of the within-subject factors (all $P$-values $>0.05$ ). Therefore, no post hoc analyses were conducted.

\section{LPC2}

Again a significant main effect for topography was found (see Table 1). However, none of the other factors (including the most relevant main effect of the factor emotion) reached significance level. Neither did we find a significant impact for the between-subject factor vigilance on LPC2 $(\mathrm{F}[1,90]=2.649 ; P=0.107)$. Therefore, no post-hoc analyses were conducted.

\section{Discussion}

The aim of the present study was to examine whether pictorial pain cues (pain-related facial expressions) embedded in a primary task paradigm can be utilized for detecting vigilance for pain using ERP protocols, as has been shown for verbal pain cues. The hypothetical background was the assumption of an outstanding salience of pain cues compared to cues for other emotional categories. Altogether, the ERP data presented here did not yield evidence that participants exhibited a particular vigilance to pictorial pain cues. This may suggest that the type of stimulus material used may be critical because of contrasting positive findings with pain words in previous studies. However, recent findings with almost identical pictorial stimuli by Gonzáles-Roldan et $\mathrm{al}^{34}$ and Reicherts et al, ${ }^{36}$ who both demonstrated enhanced LPC amplitudes, casts doubts on the assumption of generally ineffective pictorial stimulus material. Therefore, it is worth comparing these two studies with ours in detail before discussing other aspects of our results.

The stimulus material for both the Gonzáles-Roldan et $\mathrm{al}^{34}$ and the Reicherts et $\mathrm{al}^{36}$ studies, as well as of our study, originates from the Montréal pain and affective face clips, ${ }^{18}$ showing actors facially displaying emotions including pain. However, we deprived the material of two features, color and dynamics. We extracted black-and-white snapshots from the original video clips while Gonzáles-Roldan et $\mathrm{al}^{34}$ and Reicherts et $\mathrm{al}^{36}$ used the original clips. We did this because color is a potential confounder in emotion experiments, and the original was not standardized for the facial dynamics but only for the facial summary activity. However, even if we did this for good reasons, we might have reduced the emotional salience of the stimulus material. This assumption is corroborated by an observation of Gonzáles-Roldan et $\mathrm{al}^{34}$ that the shorter the video clips were - which also means the closer to a snapshot - the smaller the registered LPC modulation became. Furthermore, we presented the pain and emotional faces as task-irrelevant stimuli in a primary task procedure while Gonzáles-Roldan et $\mathrm{al}^{34}$ and Reicherts et a ${ }^{36}$ used a passive viewing paradigm. Therefore, only in our study did the stimulus material have to compete with other task requirements during trials for capturing attention. The lesson to be learned might be that even a pain face is not prioritized in any 
case but requires some emotional richness and low competition for attentional resources to be of great salience. Further studies are needed to determine, more systematically, which contextual properties of the facial display of pain favor its prioritization while processing. Certainly, there are further differences between our study and those of Gonzáles-Roldan et $\mathrm{al}^{34}$ and Reicherts et $\mathrm{al},{ }^{36}$ especially regarding the exact timing of stimulus presentation.

Our present study seems also to be inconsistent with previous investigations having used words for pain and other emotions in primary task paradigms, suggesting increased P300 and LPC activity for pain-related words in a healthy sample ${ }^{16}$ and in subclinically depressed subjects. ${ }^{15}$ One explanation might be that the visual discrimination task used in the present study absorbed more attentional resources, preventing processing of the pain and emotional faces, compared to these two studies with verbal material, where the primary task consists of a lexical decision. Another explanation, which is slightly counterintuitive, might be that pain words compare very well as regards their salience with biologically better grounded stimuli like pain faces. Brain imaging data would corroborate such an assumption. ${ }^{2}$ However, direct comparison of verbal and nonverbal pain cues for their attentional capture under otherwise identical experimental conditions is still missing.

Moreover, it is generally possible to demonstrate effects of pictorial material on ERPs as other studies, using primary task paradigms, clearly showed emotional ERP modulation by such stimuli. ${ }^{37,38}$ Since these studies did not use pain-related pictorial stimuli, perhaps our assumption of a particular salience of these cues compared to other cues of negative emotions like fear, anxiety, and anger is not justified. However, there are alternative arguments which speak in favor of the idea that facial expressions of pain are salient enough to compete well with other emotional stimuli in a primary task paradigm. Studies using the dot-probe task have found strong attentional biases towards pain utilizing pain-related and other faces in chronic pain-patients, ${ }^{12}$ as well as enhanced early engagement and later disengagement towards pain stimuli in subjects with high fear of pain levels. ${ }^{14}$ Also, research based on related paradigms suggests that increased attentional engagement occurs for threatening facial expressions, ${ }^{39,40}$ including ERP modulation. ${ }^{25,26,32,41,42}$ The outstanding role that pain-related faces may play under certain conditions was already suggested by the two studies considered in detail at the beginning of the discussion. ${ }^{34,36}$ Together, these numerous reports and our findings suggest that the facial expressions of pain may have particular salience, as long as a certain level of emotional richness (color, dynamics) is preserved. The exposure time may additionally influence the salience of pain-related stimuli. Results from a recent meta-analysis ${ }^{43}$ suggest that pain-related stimuli attract attention more effectively when presented on a clearly supraliminal level, which allows for conscious and elaborate processing, than when presented subliminally. (Our presentation time of 300 milliseconds fell between the margins for clear distinction of subliminal or supraliminal). The effect of presentation time may cause a difference to the impact of exposure time for fear and anxiety related material.

Another factor responsible for the negative findings might have been the design of the primary task in the present study as already suggested. In response to the hypothetical question of why we applied such a demanding primary task-paradigm rather than a task that absorbed less attentional resources, the answer is as follows: for adequate assessment of the level of pain vigilance, it is essential to establish a critical balance of the allocation of attention between the primary task on the one side and the concurrently presented taskirrelevant stimuli on the other side. Such a balance should guarantee that the primary task captures most of attentional resources under normal conditions, while the task-irrelevant stimuli (eg, different facial expressions of emotions) require a distinct degree of salience in order to capture attention. Pain faces were expected to do so. It is an empirical titration process to find the ideal balance, which we may still have missed. The occurrence of a high attentional load produced by the primary task can be inferred from the type of modulating effects on reaction time, P300 and LPC1. Only in the trials with a visible grid did the emotional categories seem to impact the responses, whereas in the trials without the grid, they did not succeed in doing so, as indicated by significant or close to significant interactions between the grid conditions and emotion categories. (The two stimulus conditions, grid and no grid, required discriminative responses on two separate response keys.) This may appear puzzling at first glance because a free vision on the faces without overlaid grid should have guaranteed more effect of the emotional categories and not the other way round. However, it may well be that the grid trials were without major decision uncertainty because the task-relevant stimulus is present from the very beginning, whereas in the no grid trials decision, uncertainty may prevail because subjects might have been uncertain whether the grid could still appear. In fact, no late grid presentations were programmed, and subjects were instructed that the pictures would always be presented concurrently with or without a grid, respectively. This decision uncertainty in 
half of the trials might have absorbed attentional resources, thereby preventing stronger effects of pain and emotion faces on the ERPs.

Another noteworthy finding is the increased reaction time and the enhanced P300 amplitude in those individuals who described themselves as especially vigilant to pain. These changes of reaction time and P300 amplitude were, however, not specific to any kind of emotional face expression. It might well be that hypervigilance may cause more in-depth stimulus processing in situations in which threatening stimuli occur amongst others. In accord, Snider et $\mathrm{al}^{44}$ found individuals with chronic pain, who are usually more vigilant to pain than pain-free individuals, to selectively process pain-related cues at the conscious level but not at the automatic level, which also suggests a more in-depth analysis of threatening stimuli.

There are some limitations which should be addressed in future studies. More EEG electrodes should be included, especially at more posterior positions, in order to allow the examination of additional ERP components such as P100 and early posterior negativity (EPN). Furthermore, it would be interesting to test individuals who are even more prone to attend to pain signals and who might be found among individuals awaiting acute painful experiences/procedures or suffering from chronic pain. In addition, aged individuals with a long history of pain experiences might be compared with young persons, who are almost pain-naive. However, given the strong effect of impending experimental pain on attentional biases in behavioral reaction time tests, ${ }^{43}$ such influences should be studied first.

In summary, faces expressing pain, which were presented as black and white pictures under primary task conditions, did not traceably modulate the late positive ERP components (P300, LPC) in the present study on pain-free individuals, which is inconsistent with previous findings when using verbal material as pain cues or when using similar faces as video clips in passive viewing paradigms. The reasons for the lack of positive findings were discussed, but they definitely do not pertain to small statistical power, which was rather outstanding, with 100 enrolled and 92 tested subjects. It seems that pain-related stimuli are not always of outstanding salience, but that certain characteristics (eg, type of material, emotional richness of the stimuli) have to be met in order for painrelated stimuli to be prioritized over stimuli of other emotional content. Hypervigilant individuals may tend to demonstrate in-depth processing of emotional stimuli even when the threatening stimuli are presented along with non-threatening stimuli.

\section{Acknowledgments}

This study was supported by a research grant of the Deutsche Forschungsgemeinschaft (DFG LA 685/7-1).

\section{Disclosure}

The authors report no conflicts of interest in this work.

\section{References}

1. Crombez G, van Damme S, Eccleston C. Hypervigilance to pain: an experimental and clinical analysis. Pain. 2005;116(1-2):4-7.

2. Richter M, Eck J, Straube T, Miltner WH, Weiss T. Do words hurt? Brain activation during the processing of pain-related words. Pain. 2010;148(2):198-205.

3. Lautenbacher S. Commentary to "do words hurt? Brain activation during the processing of pain words" by Richter et al. Pain. 2010;148(2): 179.

4. MacLeod C, Mathews A, Tata P. Attentional bias in emotional disorders. J Abnorm Psychol. 1986;95(1):15-20.

5. Keogh E, Ellery D, Hunt C, Hannent I. Selective attentional bias for pain-related stimuli amongst pain fearful individuals. Pain. 2001;91(1-2):91-100.

6. Keogh E, Dillon C, Georgiou G, Hunt C. Selective attentional biases for physical threat in physical anxiety sensitivity. J Anxiety Disord. 2001;15(4):299-315.

7. Asmundson GJ, Kuperos JL, Norton GR. Do patients with chronic pain selectively attend to pain-related information?: preliminary evidence for the mediating role of fear. Pain. 1997;72(1-2):27-32.

8. Asmundson GJ, Hadjistavropoulos HD. Is high fear of pain associated with attentional biases for pain-related or general threat? A categorical reanalysis. J Pain. 2007;8(1):11-18.

9. Roelofs J, Peters ML, Fassaert T, Vlaeyen JW. The role of fear of movement and injury in selective attentional processing in patients with chronic low back pain: a dot-probe evaluation. J Pain. 2005;6(5): 294-300.

10. Keogh E, Thompson T, Hannent I. Selective attentional bias, conscious awareness and the fear of pain. Pain. 2003;104(1-2):85-91.

11. Roelofs J, Peters ML, van der Zijden M, Thielen FG, Vlaeyen JW. Selective attention and avoidance of pain-related stimuli: a dot-probe evaluation in a pain-free population. J Pain. 2003;4(6):322-328.

12. Khatibi A, Dehghani M, Sharpe L, Asmundson GJ, Pouretemad H. Selective attention towards painful faces among chronic pain patients: evidence from a modified version of the dot-probe. Pain. 2009;142(1-2): $42-47$.

13. Schoth DE, Liossi C. Attentional bias toward pictorial representations of pain in individuals with chronic headache. Clin J Pain. 2010;26(3): 244-250.

14. Baum C, Schneider R, Keogh E, Lautenbacher S. Different stages in attentional processing of facial expressions of pain: a dot-probe task modification. J Pain. 2013;14(3):223-232.

15. Nikendei C, Dengler W, Wiedemann G, Pauli P. Selective processing of pain-related word stimuli in subclinical depression as indicated by event-related brain potentials. Biol Psychol. 2005;70(1):52-60.

16. Dittmar O, Krehl R, Lautenbacher S. Interrelation of self-report, behavioural and electrophysiological measures assessing threat-related information processing. Pain Res Manag. 2011;16(1):33-40.

17. Goubert L, Crombez G, Van Damme S. The role of neuroticism, pain catastrophizing and pain-related fear in vigilance to pain: a structural equations approach. Pain. 2004;107(3):234-241. 
18. Baum C, Kappesser J, Schneider R, Lautenbacher S. Does vigilance to pain make person experts in facial recognition of pain? Pain Res Manag. In press. 2013.

19. First MB, Spitzer RL, Gibbon M, Williams JBW. Structured Clinical Interview for DSM-IV-TR Axis I Disorders, Research Version, Patient Edition with psychotic screen (SCID-I/P W/PSY SCREEN). New York: Biometrics Research, New York State Psychiatric Institute; 2002.

20. Grüter T, Grüter M, Bell V, Carbon CC. Visual mental imagery in congenital prosopagnosia. Neurosci Lett. 2009;453(3):135-140.

21. Simon D, Craig KD, Miltner WH, Rainville P. Brain responses to dynamic facial expressions of pain. Pain. 2006;126(1-3):309-318.

22. Jasper, HH. The ten-twenty electrode system of the International Federation. Electroencephalogr Clin Neurophysiol. 1958;10:370-375.

23. Gratton G, Coles MG, Donchin E. A new method for off-line removal of ocular artifact. Electroencephalogr Clin Neurophysiol. 1983;55(4): 468-484.

24. Picton TW, Bentin S, Berg P, et al. Guidelines for using human eventrelated potentials to study cognition: recording standards and publication criteria. Psychophysiology. 2000;37(2):127-152.

25. Miltner WH, Trippe RH, Krieschel S, Gutberlet I, Hecht H, Weiss T. Event-related brain potentials and affective responses to threat in spider/ snake-phobic and non-phobic subjects. Int J Psychophysiol. 2005;57(1): 43-52.

26. Schupp HT, Ohman A, Junghöfer M, Weike AI, Stockburger J, Hamm AO. The facilitated processing of threatening faces: an ERP analysis. Emotion. 2004;4(2):189-200.

27. Wieser MJ, Pauli P, Reicherts P, Mühlberger A. Don’t look at me in anger! Enhanced processing of angry faces in anticipation of public speaking. Psychophysiology. 2010;47(2):271-280.

28. Kissler J, Herbert C, Winkler I, Junghöfer M. Emotion and attention in visual word processing: an ERP study. Biol Psychol. 2009;80(1): 75-83.

29. Hajcak G, Nieuwenhuis S. Reappraisal modulates the electrocortical response to unpleasant pictures. Cogn Affect Behav Neurosci. 2006;6(4): 291-297.

30. Hajcak G, Olvet DM. The persistence of attention to emotion: brain potentials during and after picture presentation. Emotion. 2008;8(2): 250-255.

31. Leutgeb V, Schäfer A, Schienle A. Late cortical positivity and cardiac responsitivity in female dental phobics when exposed to phobia-relevant pictures. Int J Psychophysiol. 2011;79(3):410-416.
32. Moser JS, Hajcak G, Bukay E, Simons RF. Intentional modulation of emotional responding to unpleasant pictures: an ERP study. Psychophysiology. 2006;43(3):292-296.

33. Herrmann MJ, Aranda D, Ellgring H, et al. Face-specific event-related potential in humans is independent from facial expression. Int $J$ Psychophysiol. 2002;45(3):241-244.

34. Gonzáles-Roldan AM, Martìnez-Jauand M, Muñoz-García MA, Sitges C, Cifre I, Montoya P. Temporal dissociation in the brain processing of pain and anger faces with different intensities of emotional expression. Pain. 2011;152(4):853-859.

35. McCracken LM. "Attention" to pain in persons with chronic pain: a behavioral approach. Behav Ther. 1997;28(2):271-284.

36. Reicherts P, Wieser MJ, Gerdes AB, et al. Electrocortical evidence for preferential processing of dynamic pain expressions compared to other emotional expressions. Pain. 2012;153(9):1959-1964.

37. Schupp HT, Stockburger J, Bublatzky F, Junghöfer M, Weike AI, Hamm AO. Explicit attention interferes with selective emotion processing in human extrastriate cortex. BMC Neuroscience. 2007;8:16.

38. Schupp HT, Stockburger J, Bublatzky F, Junghöfer M, WeikeAI, HammAO. The selective processing of emotional visual stimuli while detecting auditory targets: an ERP analysis. Brain Res. 2008;1230:168-176.

39. Mogg K, Bradley BP. Some methodological issues in assessing attentional biases for threatening faces in anxiety: a replication study using a modified version of the probe detection task. Behav Res Ther. 1999;37(6):595-604.

40. Bradley BP, Mogg K, Falla SJ, Hamilton LR. Attentional bias for threatening facial expressions in anxiety: manipulation of stimulus duration. Cogn Emot. 1998;12(6):737-753.

41. Mühlberger A, Wieser MJ, Herrmann MJ, Weyers P, Tröger C, Pauli P. Early cortical processing of natural and artificial emotional faces differs between lower and higher socially anxious persons. J Neural Transm. 2009;116(6):735-746.

42. Kolassa IT, Kolassa S, Musial F, Miltner HRW. Event-related brain potentials to schematic faces in social phobia. Cogn Emot. 2007;21(8): 1721-1744.

43. Crombez G, Van Ryckeghem DM, Eccleston C, Van Damme S. Attentional bias to pain-related information: a meta-analysis. Pain 2013;154(4):497-510.

44. SniderBS,Asmundson GJ, Wiese KC.Automatic and strategic processing of threat cues in patients with chronic pain: a modified stroop evaluation. Clin J Pain. 2000;16(2):144-154.
Journal of Pain Research

\section{Publish your work in this journal}

The Journal of Pain Research is an international, peer-reviewed, open access, online journal that welcomes laboratory and clinical findings in the fields of pain research and the prevention and management of pain. Original research, reviews, symposium reports, hypothesis formation and commentaries are all considered for publication.

\section{Dovepress}

The manuscript management system is completely online and includes a very quick and fair peer-review system, which is all easy to use. Visit http://www.dovepress.com/testimonials.php to read real quotes from published authors. 